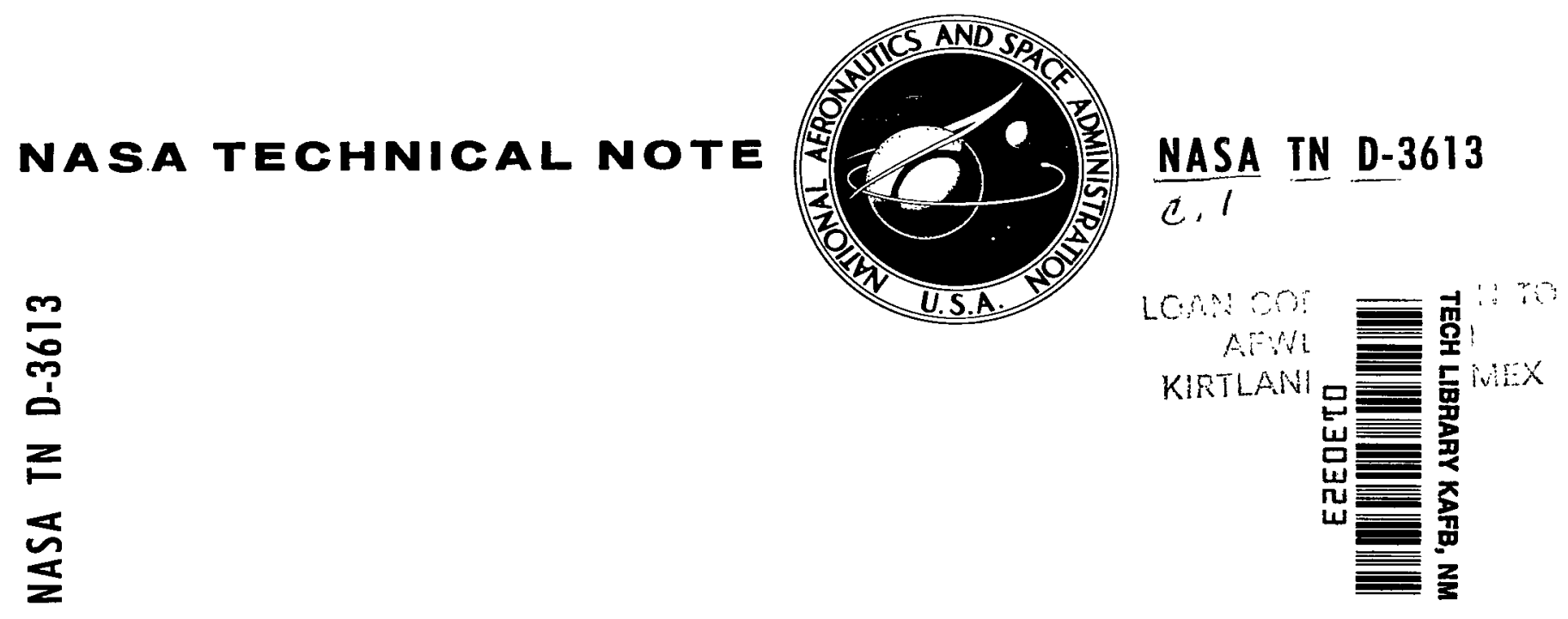

WEAK LOCALLY HOMOGENEOUS TURBULENCE IN IDEALIZED FLOW THROUGH A CONE

by Robert G. Deissler

Lewis Research Center Cleveland, Obio

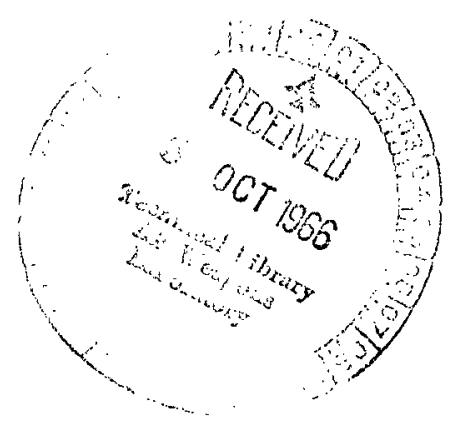

national aERONAUtics and SPACE AdMINISTRATION - WASHINGTON, D. C. - SEPTEMBER 1966 
WEAK LOCALLY HOMOGENEOUS TURBULENCE IN

\section{IDEALIZED FLOW THROUGH A CONE}

By Robert G. Deissler

Lewis Research Center

Cleveland, Ohio

NATIONAL AERONAUTICS AND SPACE ADMINISTRATION

For sale by the Clearinghouse for Federal Scientific and Technical Information Springfield, Virginia 22151 - Price $\$ 2.00$ 


\title{
WEAK LOCALLY HOMOGENEOUS TURBULENCE IN \\ IDEALIZED FLOW THROUGH A CONE
}

\author{
by Robert G. Deissler
}

Lewis Research Center

\begin{abstract}
SUMMARY
Turbulence in idealized flow through a cone is analyzed by using generalized twopoint correlation equations that are based on the Navier-Stokes and continuity equations. Viscosity and the effects of mean strain are retained in the analysis, but the turbulence is assumed to be weak enough to neglect the triple correlation terms in the equations in comparison with the other terms. Components of the turbulent velocity and vorticity variances, as well as the spectra of those quantities, are calculated. A mean-strain energy-transfer term in the spectral equation which transfers energy components between wave numbers is also considered. By using the momentum-heat-transfer analogy, the results are related to heat transfer between the fluid and the cone wall.
\end{abstract}

\section{INTRODUCTION}

When fluid flows through a section of a cone, the fluid elements are distorted because of the changing cross sectional area of the flow. If turbulence is initially present, it is modified by this distortion of the fluid. The interaction between weak turbulence and an idealized distorting mean flow in a cone is studied in this report. Flow in a cone is of particular interest because wind tunnels and rocket nozzles frequently contain conical sections.

The effect of an irrotational distortion (no shear) on turbulence has been studied in references 1 to 4 . In these studies the effects of viscosity were neglected and the distortion was assumed to occur so rapidly that the turbulent velocities have a negligible effect on the motion during distortion. The present work is more closely related to that of Pearson (ref. 5). In his work the effects of viscosity are included,and the requirement that the distortion be rapid is not imposed. If the distortion is not rapid, however, the turbulence must be weak enough in comparison with other terms in the equations to 
neglect terms containing triple correlations. Pearson's analysis assumes that the normal velocity gradients are uniform and that the turbulence is homogeneous.

The present analysis of turbulence in incompressible idealized flow through a cone uses the generalized two-point correlation equations that are based on the Navier-Stokes and continuity equations. The normal velocity gradients $\partial \mathrm{U}_{1} / \partial \mathrm{x}_{1}, \partial \mathrm{U}_{2} / \partial \mathrm{x}_{2}$ and $\partial \mathrm{U}_{3} / \partial \mathrm{x}_{3}$ are allowed to vary axially but not transversely. (Symbols are defined in the appendix.) The turbulence is assumed to be homogeneous in the transverse direction but only locally homogeneous in the axial direction. That is, the variation in the intensity of the turbulence over a correlation (or mixing) length in the axial direction is assumed to be small. The mean axial velocity is assumed uniform over a cross section, and mean shear stresses are assumed absent. The turbulence is initially isotropic but is allowed to become anisotropic under the distorting influence of the mean flow. Components of the turbulent velocity and vorticity variances are calculated, as well as components of the spectra of those quantities. A mean-strain energy-transfer term in the spectral equation which transfers energy components between wave numbers is also considered. By using the momentum-heat-transfer analogy, the results are related to heat transfer between the fluid and the cone wall. The basic equations required in the analysis will be considered in the next section.

\section{BASIC EQUATIONS}

General two-point correlation equations for turbulence in an incompressible fluid with mean velocity gradients are obtained in reference 6 from the Navier-Stokes and continuity equations as follows:

$$
\begin{aligned}
& \frac{\partial}{\partial t} \overline{u_{i} u_{j}^{q}}+\overline{u_{k} u_{j}^{\prime}} \frac{\partial U_{i}}{\partial x_{k}}+\overline{u_{i} u_{k}^{\prime}} \frac{\partial U_{j}^{\prime}}{\partial x_{k}^{\prime}}+\left(U_{k}^{\prime}-U_{k}\right) \frac{\partial}{\partial r_{k}} \overline{u_{i} u_{j}^{\prime}}+\frac{1}{2}\left(U_{k}+U_{k}^{\prime}\right) \frac{\partial}{\partial\left(x_{k}\right)_{m}} \overline{u_{i} u_{j}^{\prime}} \\
& +\frac{1}{2} \frac{\partial}{\partial\left(x_{k}\right)_{m}} \overline{\left(u_{i}^{\prime} u_{j}^{\prime} u_{k}^{\prime}\right.}+\overline{\left.u_{i} u_{k} u_{j}^{q}\right)}+\frac{\partial}{\partial r_{k}} \overline{\left(u_{i} u_{j}^{\prime} u_{k}^{\prime}\right.}-\overline{\left.u_{i} u_{k} u_{j}^{\prime}\right)} \\
& =-\frac{1}{\rho}\left\{\frac{1}{2}\left[\frac{\partial}{\partial\left(\mathrm{x}_{\mathrm{i}}\right)_{\mathrm{m}}} \overline{\mathrm{pu}_{\mathrm{j}}^{\prime}}+\frac{\partial}{\partial\left(\mathrm{x}_{\mathrm{j}}\right)_{\mathrm{m}}} \overline{\mathrm{u}_{\mathrm{i}} \mathrm{p}^{\prime}}\right]+\frac{\partial}{\partial \mathrm{r}_{\mathrm{j}}} \overline{\mathrm{u}_{\mathrm{i}} \mathrm{p}^{\prime}}-\frac{\partial}{\partial \mathrm{r}_{\mathrm{i}}} \overline{\mathrm{pu}} \overline{\mathrm{j}}\right\} \\
& +\frac{1}{2} \nu \frac{\partial^{2} \overline{u_{i} u_{j}^{\prime}}}{\partial\left(x_{k}\right)_{m} \partial\left(x_{k}\right)_{m}}+2 \nu \frac{\partial^{2} \bar{u}_{i} u_{j}^{2}}{\partial r_{k} \partial r_{k}}
\end{aligned}
$$




$$
\begin{array}{r}
\frac{1}{\rho}\left[\frac{1}{4} \frac{\partial^{2} \overline{u_{i} p^{\prime}}}{\partial\left(x_{j}\right)_{m} \partial\left(x_{j}\right)_{m}}+\frac{\partial^{2} \overline{u_{i} p^{\prime}}}{\partial\left(x_{j}\right)_{m} \partial r_{j}}+\frac{\partial^{2} \overline{u_{i} p^{p^{\prime}}}}{\partial r_{j} \partial r_{j}}\right]=-2 \frac{\partial v_{j}^{\prime}}{\partial x_{k}^{\prime}}\left[\frac{1}{2} \frac{\partial \overline{u_{i} u_{k}^{+}}}{\partial\left(x_{j}\right)_{m}}+\frac{\partial \overline{u_{i} u_{k}^{\prime}}}{\partial r_{j}}\right] \\
-\frac{1}{4} \frac{\partial^{2} \overline{u_{i} u_{j}^{p} u_{k}^{+}}}{\partial\left(x_{j}\right)_{m} \partial\left(x_{k}\right)_{m}}-\frac{1}{2} \frac{\partial^{2} \overline{u_{i} u_{j}^{\prime} u_{k}^{\prime}}}{\partial\left(x_{j}\right)_{m} \partial r_{k}}-\frac{1}{2} \frac{\partial^{2} \overline{u_{i} u_{j}^{\prime} u_{k}^{\prime}}}{\partial\left(x_{k}\right)_{m} \partial r_{j}}-\frac{\partial^{2} \overline{u_{i} u_{j}^{\prime} u_{k}^{\prime}}}{\partial r_{j} \partial r_{k}}
\end{array}
$$

and

$$
\begin{aligned}
& \frac{1}{\rho}\left[\frac{1}{4} \frac{\partial^{2} \overline{p u_{j}^{i}}}{\partial\left(x_{i}\right)_{m} \partial\left(x_{i}\right)_{m}}-\frac{\partial^{2} \overline{p u_{j}^{i}}}{\partial\left(x_{i}\right)_{m} \partial r_{i}}+\frac{\partial^{2} \overline{p u_{j}^{\prime}}}{\partial r_{i} \partial r_{i}}\right]=-2 \frac{\partial U_{i}}{\partial x_{k}}\left[\frac{1}{2} \frac{\partial \overline{u_{k} u_{j}^{i}}}{\partial\left(x_{i}\right)_{m}}-\frac{\partial \overline{u_{k} \mathbf{u}_{j}^{i}}}{\partial r_{i}}\right] \\
& -\frac{1}{4} \frac{\partial^{2} \overline{u_{i} u_{k} u_{j}^{+}}}{\partial\left(x_{i}\right)_{m} \partial\left(x_{k}\right)_{m}}+\frac{1}{2} \frac{\partial^{2} \overline{u_{i} u_{k} u_{j}^{p}}}{\partial\left(x_{i}\right)_{m} \partial r_{k}}+\frac{1}{2} \frac{\partial^{2} \overline{u_{i} u_{k} u_{j}^{p}}}{\partial\left(x_{k}\right)_{m} \partial r_{i}}-\frac{\partial^{2} \overline{u_{i} u_{k} u_{j}^{p}}}{\partial r_{i} \partial r_{k}}
\end{aligned}
$$

The vector configuration for correlations between fluctuating quantities at points $\mathbf{P}$ and $P^{r}$ is shown in figure 1 . The quantities $u_{i}$ and $u_{j}^{\prime}$ are fluctuating velocity components at $P$ and $P^{\prime}, U_{i}$ and $U_{j}^{\prime}$ are mean velocity components, $x_{i}$ is a space coordinate, $t$ is the time, $\rho$ is the density, $\nu$ is the kinematic viscosity, and $p$ is the instantaneous pressure. Bars over terms designate correlations or averaged quantities. The subscripts can take on the values 1,2 , or 3 , and a repeated subscript in a term indicates a summation.

For locally homogeneous turbulence the turbulence is considered homogeneous over

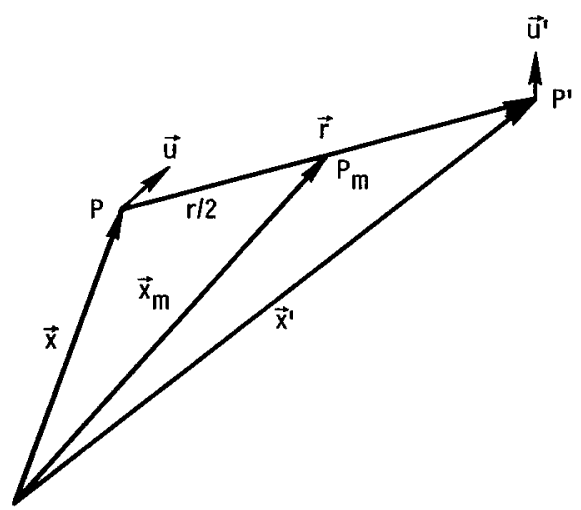

Figure 1. - Vector configuration for two-point correlation equations. a correlation length, or the scale of the inhomogeneity is much greater than the scale of the turbulence. Thus, a quantity such as $\partial^{2} \overline{u_{i} u_{j}^{i}} / \partial\left(x_{k}\right)_{m} \partial\left(x_{k}\right)_{m}$ in equation (1) will be negligible compared with $\partial^{2}{\overline{u_{i}}}_{\mathbf{j}} / \partial \mathbf{r}_{\mathbf{k}} \partial \mathbf{r}_{\mathbf{k}}$. (A calculation for axially decaying turbulence without mean velocity gradients (ref. 6, fig. 3) shows that this is a good approximation except very close to the virtual origin of the turbulence.) In general, for locally homogeneous turbulence,

$$
\frac{\partial}{\partial\left(\mathbf{x}_{\mathbf{i}}\right)_{\mathrm{m}}}<<\frac{\partial}{\partial \mathbf{r}_{\mathbf{i}}}
$$


Also, for that type of turbulence the mean velocity will vary linearly over distances for which correlations are appreciable so that

$$
\begin{gathered}
\frac{\partial U_{j}^{\prime}}{\partial x_{k}^{\prime}}=\frac{\partial U_{j}}{\partial x_{k}}=\frac{\partial\left(U_{j}\right)_{m}}{\partial\left(x_{k}\right)_{m}} \\
U_{k}^{s}-U_{k}=\frac{r_{\ell} \partial\left(U_{k}\right)_{m}}{\partial\left(x_{\ell}\right)_{m}} \\
\frac{\left(U_{k}+U_{k}^{*}\right)}{2}=\left(U_{k}\right)_{m}
\end{gathered}
$$

Finally, in order to make the set of equations determinate, the turbulence is assumed weak enough to neglect terms containing triple correlations. It should be noted that the turbulence in a flow with large velocity gradients may not have to be as weak as that in a flow without velocity gradients. The terms containing those gradients may be large compared with triple correlation terms, even if the turbulence is moderately strong. Equations (1), (2), and (3) become, for the steady state at a fixed point,

$$
\begin{aligned}
& \overline{u_{k} u_{j}^{p}} \frac{\partial\left(U_{i}\right)_{m}}{\partial\left(x_{k}\right)_{m}}+\overline{u_{i} u_{k}^{p}} \frac{\partial\left(U_{j}\right)_{m}}{\partial\left(x_{k}\right)_{m}}+\frac{\partial\left(U_{k}\right)_{m}}{\partial\left(x_{\ell}\right)_{m}} r_{\ell} \frac{\partial}{\partial r_{k}} \overline{u_{i} u_{j}^{p}}+\left(U_{k}\right)_{m} \frac{\partial}{\partial\left(x_{k}\right)_{m}} \overline{u_{i} u_{j}^{?}} \\
& =-\frac{1}{\rho}\left(\frac{\partial}{\partial \mathrm{r}_{\mathrm{j}}} \overline{\mathrm{u}_{\mathrm{i}} \mathrm{p}^{\prime}}-\frac{\partial}{\partial \mathrm{r}_{\mathrm{i}}} \overline{\mathrm{pu}_{\mathrm{j}}^{\dagger}}\right)+2 \nu \frac{\partial^{2} \overline{\mathrm{u}_{\mathrm{i}} \mathrm{u}_{\mathrm{j}}^{\dagger}}}{\partial \mathrm{r}_{\mathrm{k}} \partial \mathrm{r}_{\mathrm{k}}} \\
& \frac{1}{\rho} \frac{\partial^{2} \overline{u_{i} p^{\imath}}}{\partial r_{j} \partial r_{j}}=-2 \frac{\partial\left(U_{j}\right)_{m}}{\partial\left(x_{k}\right)_{m}} \frac{\partial \overline{u_{i} u_{k}^{i}}}{\partial r_{j}} \\
& \frac{1}{\rho} \frac{\partial^{2} \overline{\mathrm{pu}_{\mathrm{j}}^{\uparrow}}}{\partial \mathrm{r}_{\mathrm{i}} \partial \mathrm{r}_{\mathrm{i}}}=2 \frac{\partial\left(\mathrm{U}_{\mathrm{i}}\right)_{\mathrm{m}}}{\partial\left(\mathrm{x}_{\mathrm{k}}\right)_{\mathrm{m}}} \frac{\partial \overline{\mathrm{u}_{\mathrm{k}} \mathrm{u}_{\mathrm{j}}^{\dagger}}}{\partial \mathrm{r}_{\mathrm{i}}}
\end{aligned}
$$

Equations (4), (5), and (6) are the correlation equations for steady-state locally homogeneous turbulence with mean velocity gradients. The equations can be converted to spectral form by introducing the usual three-dimensional Fourier transforms defined 
as follows:

$$
\begin{aligned}
\overline{u_{i} u_{j}^{r}} & =\int_{-\infty}^{\infty} \varphi_{i j} e^{i \vec{k} \cdot \vec{r}} d \vec{\kappa} \\
\overline{p_{j}^{q}} & =\int_{-\infty}^{\infty} \lambda_{j} e^{i \vec{\kappa} \cdot \vec{r}} d \vec{\kappa} \\
\overline{u_{i} p^{2}} & =\int_{-\infty}^{\infty} \lambda_{i}^{i} e^{i \vec{k} \cdot \vec{r}} d \vec{\kappa}
\end{aligned}
$$

where $\mathrm{d} \vec{\kappa}=\mathrm{d} \kappa_{1} \mathrm{~d} \kappa_{2} \mathrm{~d} \kappa_{3} . \quad$ Then,

$$
\mathrm{r}_{\ell} \frac{\partial \overline{\mathbf{u}_{\mathbf{i}} \mathbf{u}_{\mathbf{j}}}}{\partial \mathbf{r}_{\mathbf{k}}}=\int_{-\infty}^{\infty}-\left(\kappa_{\mathrm{k}} \frac{\partial \varphi_{\mathbf{i j}}}{\partial \kappa_{\ell}}+\delta_{\ell \mathbf{k}} \varphi_{\mathbf{i j}}\right) \mathrm{e}^{\mathbf{i} \overrightarrow{\boldsymbol{k}} \cdot \overrightarrow{\mathbf{r}}} \mathrm{d} \vec{\kappa}
$$

where $\delta_{\ell k}$ is the Kronecker delta. Equation (10) can be obtained by differentiating equation (7) with respect to $r_{k}$, writing the inverse transform, and then differentiating with respect to $\kappa_{\ell}$. Taking the Fourier transforms of equations (4) to (6) results in

$\varphi_{\mathrm{kj}} \frac{\partial\left(\mathrm{U}_{\mathrm{i}}\right)_{\mathrm{m}}}{\partial\left(\mathrm{x}_{\mathrm{k}}\right)_{\mathrm{m}}}+\varphi_{\mathrm{ik}} \frac{\partial\left(\mathrm{U}_{\mathrm{j}}\right)_{\mathrm{m}}}{\partial\left(\mathrm{x}_{\mathrm{k}}\right)_{\mathrm{m}}}-\frac{\partial\left(\mathrm{U}_{\mathrm{k}}\right)_{\mathrm{m}}}{\partial\left(\mathrm{x}_{\ell}\right)_{\mathrm{m}}}\left(\kappa_{\mathrm{k}} \frac{\partial \varphi_{\mathrm{ij}}}{\partial \kappa_{\ell}}+\delta_{\ell \mathrm{k}} \varphi_{\mathbf{i j}}\right)+\left(\mathrm{U}_{\mathrm{k}}\right)_{\mathrm{m}} \frac{\partial}{\partial\left(\mathrm{x}_{\mathrm{k}}\right)_{\mathrm{m}}} \varphi_{\mathrm{ij}}$

$$
\begin{aligned}
& =-\frac{1}{\rho}\left(i \kappa_{j} \lambda_{i}^{\prime}-i \kappa_{i} \lambda_{j}\right)-2 \nu \kappa^{2} \varphi_{i j} \\
& -\frac{1}{\rho}{ }_{i} \kappa_{j} \lambda_{i}^{\prime}=2 \frac{\partial\left(\mathrm{U}_{\ell}\right)_{m}}{\partial\left(\mathrm{x}_{\mathrm{k}}\right)_{\mathrm{m}}} \frac{\kappa_{\ell} \kappa_{\mathrm{j}}}{\kappa^{2}} \varphi_{\mathrm{ik}} \\
& \left.\frac{1}{\rho} i \kappa_{i} \lambda_{j}=2 \frac{\partial\left(\mathrm{U}_{\ell}\right)_{m}}{\partial\left(\mathrm{x}_{\mathrm{k}}\right)_{\mathrm{m}}} \frac{\kappa_{\ell} \kappa_{\mathrm{i}}}{\kappa^{2}} \varphi_{\mathrm{jk}}\right\}
\end{aligned}
$$

Combining equations (11) and (12) and noting that $\delta_{\ell \mathbf{k}} \partial \mathrm{U}_{\ell} / \partial \mathrm{x}_{\mathbf{k}}=\partial \mathrm{U}_{\ell} / \partial \mathrm{x}_{\ell}=0$ by continuity result in 


$$
\begin{aligned}
\left(\mathrm{U}_{\mathrm{k}}\right)_{\mathrm{m}} \frac{\partial}{\partial\left(\mathrm{x}_{\mathrm{k}}\right)_{\mathrm{m}}} \varphi_{\mathrm{ij}}=\frac{\partial\left(\mathrm{U}_{\ell}\right)_{\mathrm{m}}}{\partial\left(\mathrm{x}_{\mathrm{k}}\right)_{\mathrm{m}}}\left[\left(2 \frac{\kappa_{\ell} \kappa_{\mathrm{j}}}{\kappa^{2}}-\delta_{\mathrm{j} \ell}\right) \varphi_{\mathrm{ik}}+\left(2 \frac{\kappa_{\ell} \kappa_{\mathrm{i}}}{\kappa^{2}}-\delta_{\mathrm{i} \ell}\right) \varphi_{\mathrm{kj}}\right. & \\
& \left.+\kappa_{\ell} \frac{\partial \varphi_{\mathrm{ij}}}{\partial \kappa_{\mathrm{k}}}\right]-2 \nu \kappa^{2} \varphi_{\mathrm{ij}}
\end{aligned}
$$

Equation (13) is the spectral equation for steady-state weak locally homogeneous turbulence with mean velocity gradients.

Consider next the case where the mean strain is irrotational, that is, where the shearing components of the mean velocity gradient are zero. Let

$$
\begin{aligned}
& \mathbf{s}_{(1)} \equiv \frac{\partial\left(U_{1}\right)_{m}}{\partial\left(x_{1}\right)_{m}} \\
& s_{(2)} \equiv \frac{\partial\left(U_{2}\right)_{m}}{\partial\left(x_{2}\right)_{m}} \\
& s_{(3)} \equiv \frac{\partial\left(U_{3}\right)_{m}}{\partial\left(x_{3}\right)_{m}}
\end{aligned}
$$

Then equation (13) becomes, for irrotational strain,

$\left(\mathrm{U}_{\mathrm{k}}\right)_{\mathrm{m}} \frac{\partial}{\partial\left(\mathrm{x}_{\mathrm{k}}\right)_{\mathrm{m}}} \varphi_{\mathrm{ij}}=\mathrm{s}_{(\ell)}\left[\left(2 \frac{\kappa_{\ell} \kappa_{\mathrm{i}}}{\kappa^{2}}-\delta_{\mathrm{i} \ell}\right) \varphi_{\mathrm{j} \ell}+\left(2 \frac{\kappa_{\ell} \kappa_{\mathrm{j}}}{\kappa^{2}}-\delta_{\mathrm{j} \ell}\right) \varphi_{\ell \mathrm{i}}\right.$

$$
\left.+\kappa_{\ell} \frac{\partial \varphi_{\mathrm{ij}}}{\partial \kappa_{\ell}}\right]-2 \nu \kappa^{2} \varphi_{\mathrm{ij}}
$$

where $(\ell)$ is not a tensor subscript. For axisymmetric strain at each point in a cross section, as occurs in uniform flow through a cone, $s_{(2)}=s_{(3)}$, and by continuity of the mean flow,

$$
\mathrm{s}_{(2)}=\mathrm{s}_{(3)}=-\left(\frac{1}{2}\right) \mathrm{s}_{(1)}
$$


The subscript 1 refers to the direction of an axis of symmetry for the turbulence. Since $s_{(1)}=f\left(x_{1}\right)$, integration of equation (15) gives $U_{2}=-(1 / 2) s(1)^{x_{2}}$ and $U_{3}=-(1 / 2) s(1)^{x_{3}}$, or, for a circular cross section, the radial velocity $U_{r}=-(1 / 2) s(1)^{r}$. In addition, it is assumed that the turbulence is homogeneous over a cross section of the flow and that the turbulence changes only in the axial or $x_{1}$ direction, so that

$$
\left(\mathrm{U}_{\mathrm{k}}\right)_{\mathrm{m}} \frac{\partial \varphi_{\mathbf{i j}}}{\partial\left(\mathrm{x}_{\mathrm{k}}\right)_{\mathrm{m}}}=\left(\mathrm{U}_{\mathbf{1}}\right)_{\mathrm{m}} \frac{\partial \varphi_{\mathbf{i j}}}{\partial\left(\mathrm{x}_{1}\right)_{\mathrm{m}}}
$$

To simplify the notation, let $\left(U_{1}\right)_{m} \equiv U,\left(x_{1}\right)_{m}=x$, and $s_{(1)}=s$ in the remainder of this report. Then for $\varphi_{11}$ equation (14) becomes

$$
\frac{\mathrm{U}}{\mathrm{s}} \frac{\partial \varphi_{11}}{\partial \mathrm{x}}-\kappa_{1} \frac{\partial \varphi_{11}}{\partial \kappa_{1}}+\frac{1}{2} \kappa_{2} \frac{\partial \varphi_{11}}{\partial \kappa_{2}}+\frac{1}{2} \kappa_{3} \frac{\partial \varphi_{11}}{\partial \kappa_{3}}=\varphi_{11}\left(6 \frac{\kappa_{1} 2}{\kappa^{2}}-2-\frac{2 \nu}{\mathrm{s}} \kappa^{2}\right)
$$

where use was made of the continuity relation in the form $\kappa_{2} \varphi_{12}+\kappa_{3} \varphi_{13}=-\kappa_{1} \varphi_{11}$. Similarly, for $\varphi_{\mathrm{ij}}$,

$$
\frac{\mathrm{U}}{\mathrm{s}} \frac{\partial \varphi_{\mathrm{ii}}}{\partial \mathrm{x}}-\kappa_{1} \frac{\partial \varphi_{\mathrm{ii}}}{\partial \kappa_{1}}+\frac{1}{2} \kappa_{2} \frac{\partial \varphi_{\mathrm{ii}}}{\partial \kappa_{2}}+\frac{1}{2} \kappa_{3} \frac{\partial \varphi_{\mathrm{ii}}}{\partial \kappa_{3}}=-3 \varphi_{11}+\varphi_{\mathrm{ii}}-2 \frac{\nu}{\mathrm{s}} \kappa^{2} \varphi_{\mathrm{ii}}
$$

Next determine $U$ and $s$ as functions of $x$ for flow through a cone of arbitrary cross section. With the aid of the diagram in figure 2 these are obtained as

$$
\mathrm{U}=\mathrm{U}_{0}\left(1+\frac{\mathrm{x}-\mathrm{x}_{0}}{\mathrm{r}_{0}} \tan \alpha\right)^{-2}=\frac{\mathrm{U}_{0} \mathrm{r}_{0}^{2}}{\mathrm{x}^{2} \tan ^{2} \alpha}
$$

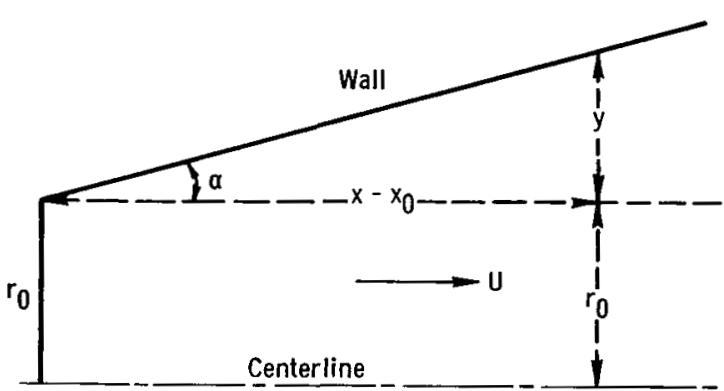

Figure 2. - Section of cone considered in analysis. Flow cross section is arbitrary; a can be positive or negative.

$$
\begin{aligned}
s & =-\frac{2 U_{0} \tan \alpha}{r_{0}}\left(1+\frac{x-x_{0}}{r_{0}} \tan \alpha\right)^{-3} \\
& =-\frac{2 U_{0} r_{0}^{2}}{x^{3} \tan ^{2} \alpha}
\end{aligned}
$$

where 


$$
\mathrm{x} \equiv \mathrm{x}-\mathrm{x}_{0}+\frac{\mathrm{r}_{0}}{\tan \alpha}=-2 \frac{\mathrm{U}}{\mathrm{s}}
$$

Then equations (17) and (18) become

$\mathrm{X} \frac{\partial \varphi_{11}}{\partial \mathrm{X}}+2 \kappa_{1} \frac{\partial \varphi_{11}}{\partial \kappa_{1}}-\kappa_{2} \frac{\partial \varphi_{11}}{\partial \kappa_{2}}-\kappa_{3} \frac{\partial \varphi_{11}}{\partial \kappa_{3}}=\left(-12 \frac{\kappa_{1}^{2}}{\kappa^{2}}+4-\frac{2 \nu \tan ^{2} \alpha}{\mathrm{U}_{0} \mathrm{r}_{0}^{2}} \mathrm{X}^{3} \kappa^{2}\right) \varphi_{11}$

and

$\mathrm{X} \frac{\partial \varphi_{\mathrm{ii}}}{\partial \mathrm{X}}+2 \kappa_{1} \frac{\partial \varphi_{\mathrm{ii}}}{\partial \kappa_{1}}-\kappa_{2} \frac{\partial \varphi_{\mathrm{ii}}}{\partial \kappa_{2}}-\kappa_{3} \frac{\partial \varphi_{\mathrm{ii}}}{\partial \kappa_{3}}=-2\left(1+\frac{\nu \tan ^{2} \alpha}{\mathrm{U}_{0} \mathrm{r}_{0}^{2}} \mathrm{X}^{3} \kappa^{2}\right) \varphi_{\mathrm{ii}}+6 \varphi_{11}$

Equations (22) and (23) apply to either diverging or converging flow through a cone, depending on whether $\alpha$ is positive or negative.

\section{SOLUTIONS OF SPECTRAL EQUATIONS}

Before equations (22) and (23) can be solved in an initial value problem, the turbulence must, of course, be specified at an initial position. It is assumed that the turbulence is isotropic at $\mathrm{x}=\mathrm{x}_{0}$, the virtual origin of the turbulence (or at $\mathrm{X}=\mathrm{x}_{0}=\mathrm{r}_{0} / \tan \alpha$, by eq. (21)), and that

$$
\left(\varphi_{\mathrm{ij}}\right)_{0}=\frac{\mathrm{J}_{0}}{12 \pi^{2}}\left(\kappa^{2} \delta_{\mathrm{ij}}-\kappa_{\mathrm{i}} \kappa_{\mathrm{j}}\right)
$$

where $J_{0}$ is a constant (ref. 6, eq. (43)).

It appears that the use of equation (24) is reasonable because it is the simplest condition that gives results that, at all values of $\mathrm{x}$, reduce to those for isotropic turbulence as the mean strain goes to zero. The use of that initial condition implies that Pearson's parameter $\tau=\nu \kappa_{0}^{2} / \mathrm{s}$ approaches $\infty$, where $\kappa_{0}$ is a characteristic initial wave number of the turbulence (ref. 5). Thus, the present results should be applicable for large kinematic viscosity, small initial turbulence scale, or small strain rate. The case $\tau \rightarrow \infty$ was not considered by Pearson.

Equations (22) and (23) are first-order partial differential equations in four inde- 
pendent variables and can be solved by methods similar to those given in reference 7 . Solutions of these equations in dimensionless form, subject to the initial condition given in equation (24), are

$$
\varphi_{11}^{*}=\frac{1}{12 \pi^{2}} \mathrm{X}^{* 6}\left(\kappa_{2}^{* 2}+\kappa_{3}^{* 2}\right) \frac{1}{\kappa^{* 4}}\left(\mathrm{X}^{*-6} \kappa_{1}^{* 2}+\kappa_{2}^{* 2}+\kappa_{3}^{* 2}\right)^{2} \mathrm{e}^{\mathrm{z}}
$$

and

$\varphi_{\mathrm{ii}}^{*}=-\frac{\kappa^{* 2}}{\kappa_{1}^{* 2}} \varphi_{11}^{*}+\frac{1}{12 \pi^{2}} \frac{\mathrm{X}^{* 6}}{\kappa_{1}^{* 2}}\left(2 \mathrm{X}^{*-6} \kappa_{1}^{* 2}+\kappa_{2}^{* 2}+\kappa_{3}^{* 2}\right)\left(\mathrm{X}^{*-6} \kappa_{1}^{* 2}+\kappa_{2}^{* 2}+\kappa_{3}^{* 2}\right) \cdot \mathrm{e}^{\mathrm{z}}$

where

$$
\begin{gathered}
\mathrm{z}=-2 \mathrm{X}^{* 2}\left[\frac{1}{7} \kappa_{1}^{* 2} \frac{\mathrm{x}^{* 7}-1}{\mathrm{x}^{* 6}\left(\mathrm{x}^{*}-1\right)}+\kappa_{2}^{* 2}+\kappa_{3}^{* 2}\right] \\
\mathrm{X}^{*}=\frac{\mathrm{x}}{\mathrm{x}_{0}}=1+\left(\mathrm{x}-\mathrm{x}_{0}\right) \frac{\tan \alpha}{\mathrm{r}_{0}} \\
\kappa_{\mathrm{i}}^{*}=\left[\frac{\nu\left(\mathrm{x}-\mathrm{x}_{0}\right)}{\mathrm{U}_{0}}\right]^{1 / 2} \kappa_{\mathrm{i}} \\
\varphi_{\mathrm{ij}}^{*}=\frac{\left(\mathrm{x}-\mathrm{x}_{0}\right) \nu}{\mathrm{J}_{0} \mathrm{U}_{0}} \varphi_{\mathrm{ij}}
\end{gathered}
$$

It is of interest that $\varphi_{11}^{*}$ and $\varphi_{i i}^{*}$ are functions only of $\left(\mathrm{x}-\mathrm{x}_{0}\right) \tan \alpha / \mathrm{r}_{0}$ and $\kappa_{\mathrm{i}}^{*}$. Because of axial symmetry it is not necessary to obtain equations for $\varphi_{22}$ and $\varphi_{33}$.

In order to integrate over wave-number space, it is convenient to introduce spherical coordinates as follows:

$$
\left.\begin{array}{c}
\kappa_{1}=\kappa \cos \theta \\
\kappa_{2}=\kappa \cos \varphi \sin \theta \\
\kappa_{3}=\kappa \sin \varphi \sin \theta
\end{array}\right\}
$$


Equation (7) then becomes, for $\overrightarrow{\mathrm{r}}=0$,

$$
\overline{u_{i} u_{j}}=\int_{0}^{\infty} \psi_{i j} d \kappa
$$

where

$$
\psi_{\mathrm{ij}}=\int_{0}^{\pi} \int_{0}^{2 \pi} \varphi_{\mathrm{ij}} \kappa^{2} \sin \theta \mathrm{d} \varphi \mathrm{d} \theta
$$

The quantity $\psi_{\mathrm{ij}}$ is a function only of the magnitude of the wave number $\kappa$ and represents an energy spectrum tensor which has been integrated over all directions in wave-number space.

The velocity variances $\overline{u_{1}^{2}}, \overline{u_{i} u_{i}}, \overline{u_{2}^{2}}$, and $\overline{u_{3}^{2}}$ are calculated from equations (25) to (30) as

$$
\begin{aligned}
& \bar{u}_{1}^{2}=\frac{1}{24 \sqrt{\pi} \mathrm{X}^{* 6}}\left\{\frac{1}{2^{3 / 2} \mathrm{X}^{* 5} \mathrm{hg}^{1 / 2}}\left(\frac{1+\mathrm{X}^{* 6}-2 \mathrm{X}^{* 12}}{\hbar}+\frac{\mathrm{X}^{* 12}}{2 \mathrm{~g}}\right)\right. \\
& +\frac{1}{2^{5 / 2} X^{* 5}(1+\hbar)^{1 / 2}}\left[\frac{2 X^{* 6}-3 X^{* 12}}{\hbar}+\frac{X^{* 12}(3+2 \hbar)}{1+\hbar}\right] \\
& \left.+\frac{3\left(1-4 \mathrm{X}^{*}+3 \mathrm{X}^{* 12}\right)}{4 \mathrm{X}^{*} \hbar^{2}} \mathrm{H}+\frac{15\left(1-2 \mathrm{X}^{* 6}+\mathrm{X}^{* 12}\right)}{8 \mathrm{X}^{* 6} \mathrm{\hbar}^{3}}\left(-\sqrt{2} \mathrm{X}^{*} \mathrm{~g}^{1 / 2}+\mathrm{B}\right)\right\} \\
& \overline{\mathrm{u}_{\mathrm{i}} \mathrm{u}_{\mathrm{i}}} *=-\frac{7^{3 / 2}}{96 \sqrt{2 \pi} \mathrm{X}^{* 2} \hbar}\left(\mathrm{X}^{*}-1\right)\left(\frac{\mathrm{X}^{*}-1}{\mathrm{X}^{* 7}-1}\right)^{1 / 2}\left[\frac{2-\mathrm{X}^{* 6}-\mathrm{X}^{* 12}}{\mathrm{X}^{* 7}-1}+\frac{3\left(1-\mathrm{X}^{* 6}\right)^{2}}{7 \hbar \mathrm{X}^{* 6}\left(\mathrm{X}^{*}-1\right)}\right] \\
& +\frac{1}{24 \sqrt{\pi} \mathrm{X}^{* 6}}\left[\frac{\mathrm{X}^{*}\left(1+\mathrm{X}^{* 6}\right)(3+2 \hbar)}{2^{5 / 2}(1+\hbar)^{3 / 2}}+\frac{1+\mathrm{X}^{* 6}-2 \mathrm{X}^{* 12}}{2^{5 / 2} \mathrm{X}^{* 5} \hbar(1+\hbar)^{1 / 2}}+\frac{3\left(1-\mathrm{X}^{* 6}\right)^{2}}{4 \mathrm{X}^{*} \hbar^{2}} \mathrm{H}\right] \\
& \overline{u_{2}^{2}}=\overline{u_{3}^{2}}=\frac{1}{2}\left(\overline{u_{i} u_{i}^{*}}-\overline{u_{1}^{2}}\right)
\end{aligned}
$$

where 


$$
\left.\begin{array}{c}
\overline{u_{i} u_{j}} *=\frac{\left(x-x_{0}\right)^{5 / 2} \nu^{5 / 2}}{J_{0} U_{0}^{5 / 2}} \overline{u_{i} u_{j}} \\
\hbar=\frac{-6 X^{* 7}+7 X^{* 6}-1}{7 X^{*}\left(X^{*}-1\right)} \\
g=\frac{X^{* 7}-1}{7 X^{*}\left(X^{*}-1\right)} \\
H=\frac{1}{X^{*} \sqrt{2 \hbar}} \ln (\sqrt{\hbar}+\sqrt{\hbar+1}) \text { for } \hbar>0 \\
H=\frac{1}{X^{*} \sqrt{-2 \hbar}} \sin ^{-1} \sqrt{-\hbar} \text { for } \hbar<0 \\
B=\frac{1}{\sqrt{2}} X^{*} \sqrt{\hbar+1}+\frac{X^{*}}{\sqrt{2 \hbar}} \ln (\sqrt{\hbar}+\sqrt{\hbar+1}) \text { for } \hbar>0 \\
B=\frac{1}{\sqrt{2}} X^{*} \sqrt{\hbar+1}+\frac{X^{*}}{\sqrt{-2 \hbar}} \sin ^{-1} \sqrt{-\hbar} \text { for } \hbar<0
\end{array}\right\}
$$

and $\mathrm{X}^{*}$ is given by equation (28).

An important physical quantity related to the turbulence is the vorticity tensor $\overline{\omega_{i} \omega_{j}}$. The vorticity spectrum tensor is given in reference 8 as

$$
\Omega_{\mathrm{ij}}=\left(\delta_{\mathrm{ij}} \kappa^{2}-\kappa_{\mathrm{i}} \kappa_{\mathrm{j}}\right) \varphi_{l \ell}-\kappa^{2} \varphi_{\mathrm{ij}}
$$

By analogy with equation (33) a directionally integrated vorticity spectrum tensor can be defined as

$$
\Lambda_{\mathrm{ij}}=\int_{0}^{\pi} \int_{0}^{2 \pi} \Omega_{\mathrm{ij}} \kappa^{2} \sin \theta \mathrm{d} \varphi \mathrm{d} \theta
$$

Then the vorticity tensor is given by 


$$
\overline{\omega_{i} \omega_{j}}=\int_{0}^{\infty} \Lambda_{i j} d \kappa
$$

One other quantity of considerable interest is the transfer term in equation (11)

$$
\frac{\partial\left(\mathrm{U}_{\mathbf{k}}\right)_{\mathrm{m}}}{\partial\left(\mathrm{x}_{\ell}\right)_{\mathrm{m}}}\left(\kappa_{\mathrm{k}} \frac{\partial \varphi_{\mathbf{i j}}}{\partial \kappa_{\ell}}+\delta_{\ell \mathbf{k}} \varphi_{\mathbf{i j}}\right)=\beta_{\mathbf{i j}}
$$

This term is, of course, nonzero only in the presence of mean strain. The term was previously discussed in references 6 and 9 for the case of a shear flow. That it can be interpreted as a transfer term can be seen from equation (10), where, if $\overrightarrow{\mathbf{r}}=0$,

$$
\int_{-\infty}^{\infty}\left(\kappa_{\mathbf{k}} \frac{\partial \varphi_{\mathbf{i j}}}{\partial \kappa_{\ell}}+\delta_{\ell \mathbf{k}} \varphi_{\mathbf{i j}}\right) \mathrm{d} \vec{\kappa}=0
$$

That is, when the term is integrated over all wave-number space it gives zero contribution to the rate of change of $\overline{u_{i} u_{j}}$. (The quantity $\left(U_{k}\right)_{m} \overline{\partial u_{i} u_{j}} / \partial\left(x_{k}\right)_{m}$ in eq. (4) can be interpreted as a rate of change.) The term $\beta_{\mathbf{i j}}$ can, however, transfer energy between wave numbers. Evidently the transfer takes place by the stretching or compressing of the vortex lines associated with the turbulence by the mean strain. This transfer is similar to that produced by triple correlations, with the exception that in that case the stretching or compressing of the vortex lines is accomplished by the action of the turbulence on itself, rather than by a mean strain. For the present case of axially symmetric irrotational strain, equation (45) becomes, for $i=j=1$,

$$
\beta_{11}=\mathbf{s}\left(\kappa_{1} \frac{\partial \varphi_{11}}{\partial \kappa_{1}}-\frac{1}{2} \kappa_{2} \frac{\partial \varphi_{11}}{\partial \kappa_{2}}-\frac{1}{2} \kappa_{3} \frac{\partial \varphi_{11}}{\partial \kappa_{3}}\right)
$$

where $\varphi_{11}$ is obtained from equation (25). As in the case of $\varphi_{i j}$ (eq. (33)), $\beta_{i j}$ can be integrated over all directions in wave-number space to give

$$
\mathrm{T}_{\mathrm{ij}}=\int_{0}^{\pi} \int_{0}^{2 \pi} \beta_{\mathrm{ij}} \kappa^{2} \sin \theta \mathrm{d} \varphi \mathrm{d} \theta
$$

Computed velocity variances, vorticities, and spectra will be discussed in the next section. 


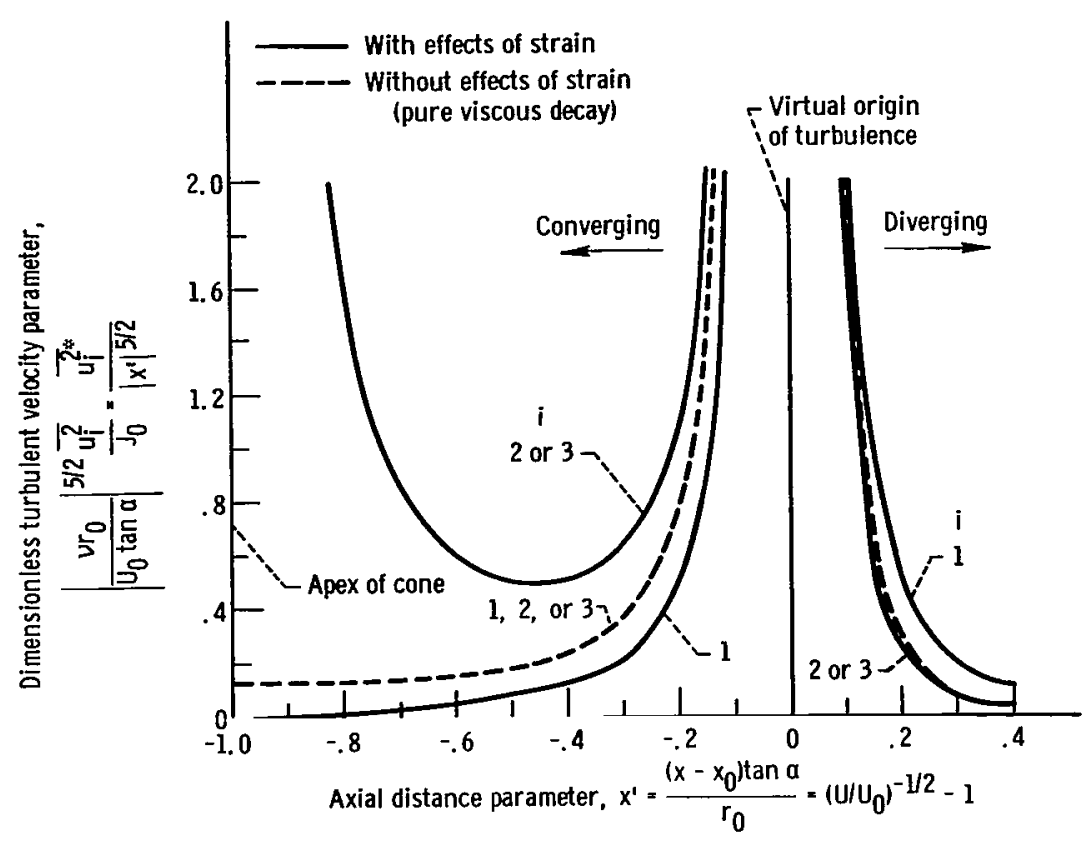

Figure 3. - Variance of turbulent velocity components for flow through cone.

\section{RESULTS AND DISCUSSION}

\section{Velocity and Vorticity Variances}

Turbulent velocity variances $\overline{u_{1}^{2}}, \overline{u_{2}^{2}}$, and $\overline{u_{3}^{2}}$, calculated using equations (34), (35), and $(36)$, are plotted in dimensionless form in figure 3 . In the ordinate, $\mathbf{u}_{i}^{2^{*}}$ is divided by $x^{\prime} 5 / 2$ in order to give quantities which are independent of $x$. Also included is the curve obtained by solving equation (22) as though the effects of strain were absent by omitting the transfer, pressure, and production terms (terms 2 to 6). The resulting equation is

$$
\left|\frac{\nu r_{0}}{\mathrm{U}_{0} \tan \alpha}\right|^{5 / 2} \frac{\overline{\mathrm{u}_{1}^{2}}}{\mathrm{~J}_{0}}=\frac{3}{16 \sqrt{2 \pi / 3}}\left|\mathrm{x}^{* 3}-1\right|^{-5 / 2}
$$

Negative values of $x^{\prime}$, the abscissa, correspond to a converging flow and positive values to a diverging flow. The virtual origin of the turbulence $\left(x^{\prime}=0\right)$ is the point at which the energy of the turbulence would be infinite.

In a converging flow, velocity fluctuations decrease from the virtual origin toward 
the apex of the cone because of viscous dissipation. The distorting influence of the cone causes the longitudinal component to decrease more rapidly and the transverse components to decrease less rapidly than they would without the effects of strain. As the apex of the cone is approached, the longitudinal component continues to decrease rapidly toward zero. The transverse components, on the other hand, begin to increase as the effects of strain become greater than the effects of viscous dissipation. From equation (20) it can be seen that the strain rate increases rapidly as the apex is approached. At the apex, where the mean velocity and strain rate approach infinity, the transverse velocity fluctuations would also become infinite. In practice, of course, the tip of the cone would have to be removed in order to allow flow. It is of interest that this increase in transverse turbulent velocity component with mean velocity or contraction ratio at large velocity ratios was not observed in reference 5 , where the velocity gradients were uniform.

For a diverging flow in a cone (positive $x^{\prime}$ ) the effects of strain are opposite to those for a converging flow. The longitudinal component decreases less rapidly than it would for no strain. Although the effect of strain is to increase the longitudinal component, that component continues to decrease as $x^{\prime}$ increases because, as shown in equation (20), the strain rate decreases with $x^{*}$. That is, the effect of viscous decay in this case is greater than the effect of strain.

Trends similar to those shown in figure 3 for converging flow have been observed experimentally (refs. 10 and 11). A comparison between the present analysis and experimental results from reference 10 for a 4 -to-1 contraction is given in figure 4 . The subscript a refers to conditions upstream of the contraction at the point where the mean velocity begins to vary. (It should be noted that the contraction in the experiment was evidently not a true cone.) Values of $\mathrm{U} / \mathrm{U}_{\mathrm{a}}$ for the analytical curves were obtained by

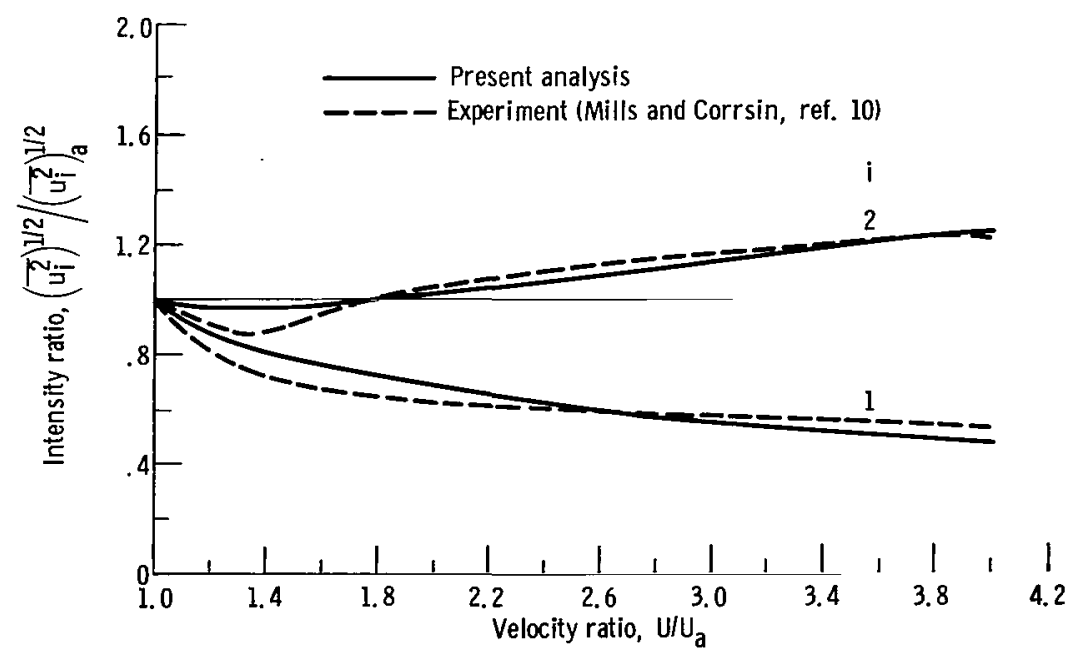

Figure 4. - Present analysis compared with experiment for converging flow. 


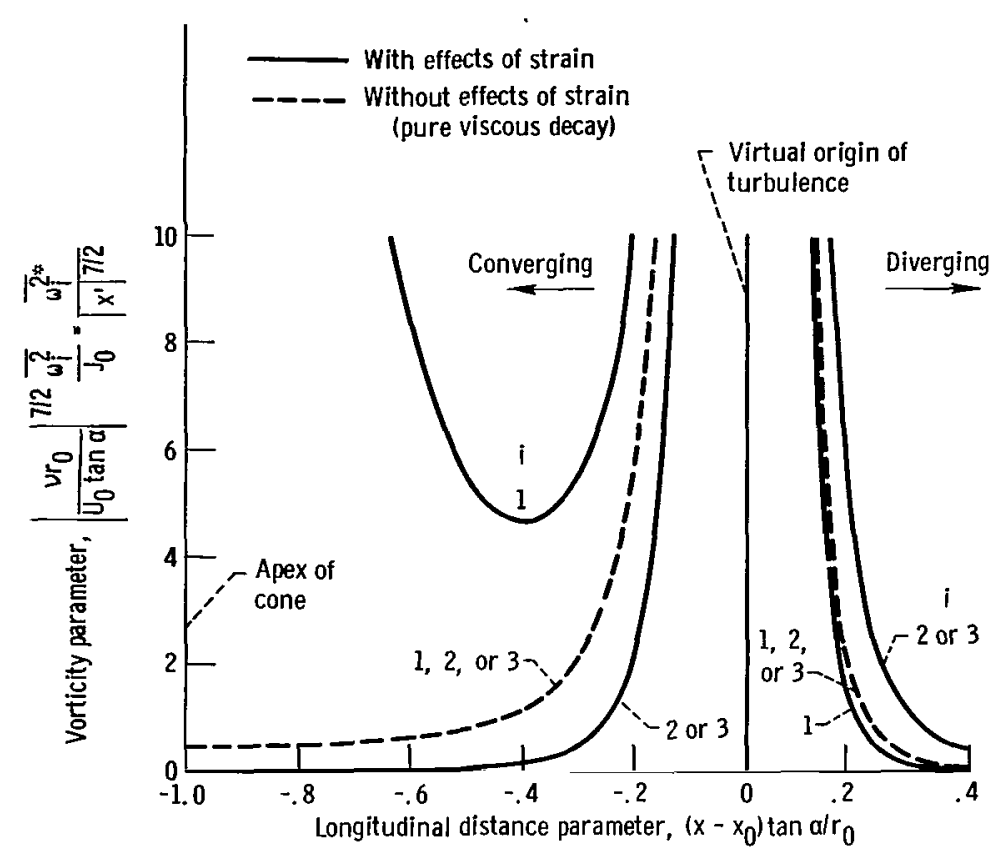

Figure 5. - Variance of furbulent vorticity components for flow through cone.

assuming that the value of $\mathrm{U} / \mathrm{U}_{\mathrm{a}}$ at the minimum point in the analytical curve for the transverse component $(i=2)$ corresponds to the value of $U / U_{a}$ at the minimum point in the experimental curve for the same component. The overall change in the turbulent components produced by the contraction appears to be given reasonably well by the analysis, but the minimum in the experimental curve for $i=2$ is sharper than that in the corresponding analytical curve.

To explain the trends shown in figure 3 , turbulent vorticity variances are plotted in figure 5. The dashed curves in the figure, for no effects of strain, were obtained from the equation

$$
\left|\frac{\nu \mathrm{r}_{0}}{\mathrm{U}_{0} \tan \alpha}\right|^{7 / 2} \frac{\overline{\omega_{1}^{2}}}{\mathrm{~J}_{0}}=\frac{45}{64 \sqrt{2 \pi / 3}}\left|\mathrm{x}^{* 3}-1\right|^{-7 / 2}
$$

In general, the trends for the vorticity components are opposite to those for the velocities. For a converging flow, the longitudinal vortices are stretched and thus strenghened by the accelerating flow and the transverse vortices are shortened along their axes and are thus weakened. The straining action also tends to turn the axes of the oblique vortices and to aline them in the direction of the cone axis. Both of these effects tend to increase the longitudinal vorticity component and decrease the transverse component, 


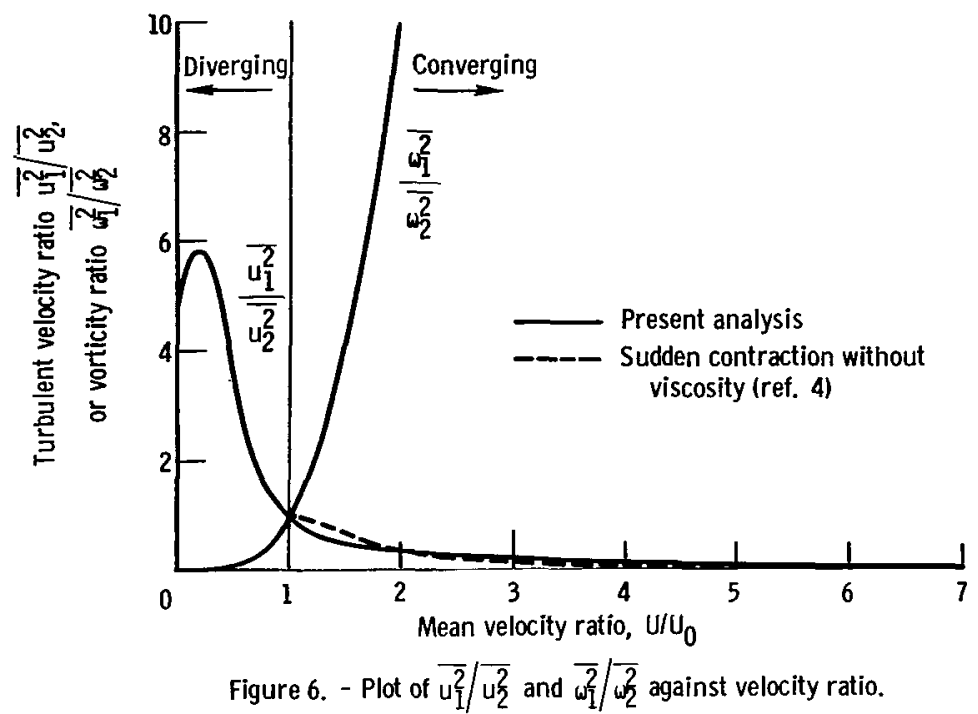

as shown in figure 5. The opposite trends occur for a diverging flow; the strain in that case tends to decrease the longitudinal vorticity component and to increase the transverse component.

The relation of the vorticity to the fluctuating velocity components for a contracting flow has been pointed out by Prandtl (ref. 1) and Taylor (ref. 2). When the vortex axes lie predominately in the direction of the mean flow, the longitudinal velocity fluctuations are small, whereas the transverse velocity fluctuations can be large. The opposite effects occur for a diverging flow. There is, however, an important difference between the two cases. This is illustrated in figure 6 , where $\overline{u_{1}^{2}} / \mathrm{u}_{2}^{2}$ and $\overline{\omega_{1}^{2}} \sqrt{\omega_{2}^{2}}$ are plotted against $\mathrm{U} / \mathrm{U}_{0}$. (The relation between this abscissa and the one in the preceding plots is given by eq. (19). ) Although $\overline{u_{1}^{2}} / \mathrm{u}_{2}^{2}$ goes to zero as $\overline{\omega_{1}^{2}} / \omega_{2}^{2}$ goes to infinity for a converging flow, the opposite limits are not approached for a diverging flow; that is, $\overline{u_{1}^{2}} \sqrt{\mathbf{u}_{2}^{2}}$ does not go to infinity as $\overline{\omega_{1}^{2}} / \overline{\omega_{2}^{2}}$ goes to zero but approaches a limiting value of 5. This occurs in diverging flow because transverse as well as longitudinal velocity fluctuations are present when the vortex axes are alined transversely, while in converging flow, the longitudinal velocity fluctuations approach zero as the vortex axes become alined longitudinally. Included in the plot (fig. 6) for comparison is the curve for $\overline{u_{1}^{2} / u_{2}^{2}}$ for a sudden contraction without viscosity as obtained from reference 4.

Relative intensity ratios for turbulent components corrected for viscous decay are plotted in figure 7. To obtain the ordinates in this figure, the ordinates for the solid curves in figure 3 were corrected to eliminate decay by dividing them by the ordinates for the dashed curves, which are for a pure viscous decay. The result, after the square root 


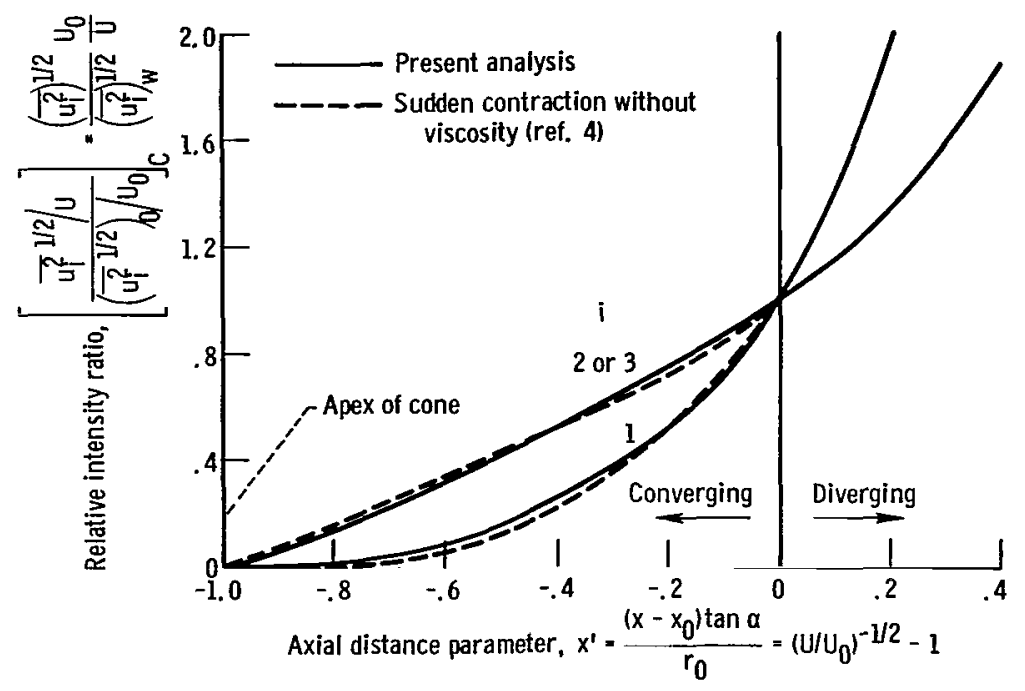

Figure 7. - Relative intensity ratios for turbulent components corrected to eliminate decay.

has been extracted, is divided by $\mathrm{U} / \mathrm{U}_{0}$ to give intensity ratios relative to the local mean velocity. Curves obtained from reference 4 for a sudden contraction and no viscosity are included in the plot in figure 7 and show fair agreement with the present analysis.

The curve in figure 7 for $\left\{\left({\overline{u_{2}^{2}}}^{1 / 2} / U\right) /\left[\left(\bar{u}_{2}^{2}\right)_{0}^{1 / 2} / U_{0}\right]\right\}$ can be related approximately to the heat transfer between a cone wall and a fluid, which occurs, for instance, in a cooled rocket nozzle. The comparison is made by using the following argument, which is based on the momentum-heat-transfer analogy: Except very close to the wall, the turbulent heat transfer is large compared with the molecular heat transfer, so that the total radial heat flow per unit area $q$ is approximately $\rho c_{p} \epsilon d T / d r$. The radial temperature gradient $\mathrm{dT} / \mathrm{dr}$ at a particular radius is assumed proportional to $\Delta \mathrm{T} / \delta$, where $\delta$ is the boundary layer thickness. The eddy diffusivity $\epsilon$ is replaced by the product of a transverse velocity fluctuation and a mixing length which is assumed proportional to $\delta$, so that $\epsilon \sim{\overline{u_{2}^{2}}}^{1 / 2} \delta$. Then the heat-transfer coefficient $\mathrm{h}=\mathrm{q} / \Delta \mathrm{T} \sim \rho \mathrm{c}_{\mathrm{p}} \overline{\mathrm{u}}^{2}{ }^{1 / 2}$, or the Stanton number $S t=h / \rho U c_{p} \sim \bar{u}_{2}^{2} / U$.

It is assumed that the change in turbulent intensity along the cone in the boundary layer is determined primarily by the normal strain rather than by the shear, as it might be when the strain is very rapid. (The initial turbulent intensity in the boundary layer at the entrance of the cone would, of course, be determined by the shear in the upstream boundary layer.) By using the preceding relation for Stanton number, the ordinate curve for $i=2$ in figure 7 can then be replaced by $S t / S t_{0}$. That curve, which has been 


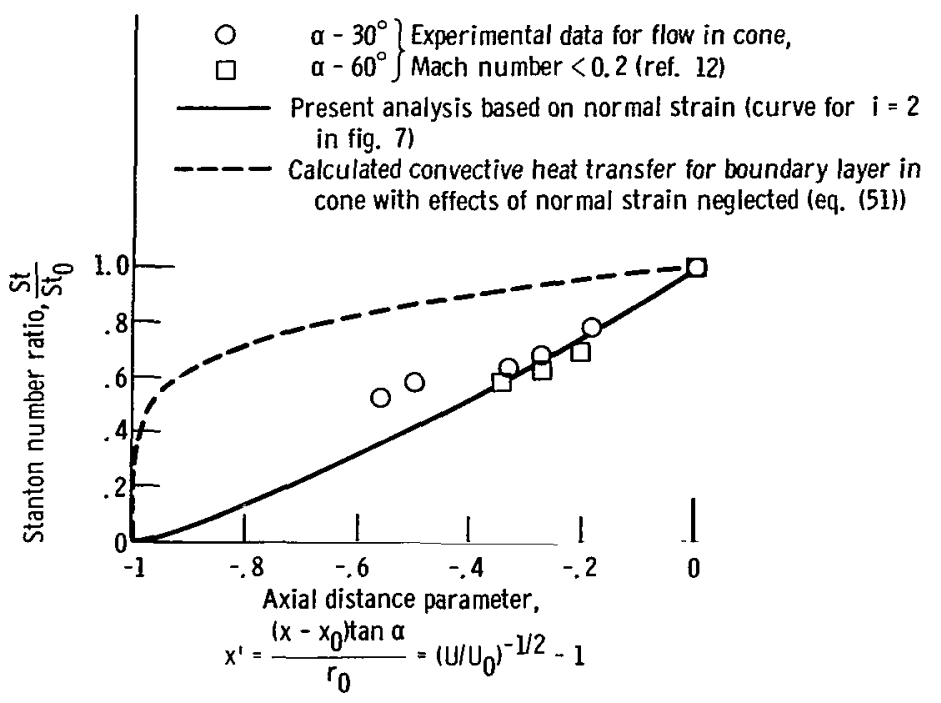

Figure 8. - Approximate calculation of heat transfer between cone wall and fluid and comparison with experiment. Angle, a degree, in figure is angle between cone generator and flow direction.

corrected to eliminate viscous decay, is used because the inhomogeneous shear which occurs in a boundary layer will normally offset the viscous decay. The curve, replotted in figure 8, shows that Stanton number decreases rapidly along a converging cone. If, by contrast, the Stanton number is calculated from local conditions in a boundary layer without considering normal strain, the decrease is much more gradual. In that case the Stanton number is roughly proportional to ( $\rho \mathrm{UD})^{-0.2}$ (assuming that $\delta \sim \mathrm{D}$ ), and

$$
\mathrm{St} / \mathrm{St}_{0}=\left(1+\mathrm{x}^{\mathrm{r}}\right)^{0.2}
$$

The curve for equation (51) is the dashed curve in figure 8.

The heat transfer in the boundary layer of a rocket nozzle may, of course, be more complicated than the case considered herein, where changes are assumed to be governed by the normal strain. The effects of shear and variable properties, as well as of normal strain, may not be negligible. However, experimental data for heat transfer in cooled conical nozzles (refs. 12 and 13) indicate trends very similar to those obtained herein. Data from reference 12 for $M<0.2$ in the conical portions of two nozzles are plotted in figure 8. Comparison of the data with the solid and dashed curves seems to indicate that changes in Stanton number along the cone are much more dependent on the normal strain than on the shear in the boundary layer. When the data were plotted, it was assumed that the entrance of the cone corresponds to $\mathrm{x}^{*}=0$. Although there is some uncertainty as to the point in the analysis which corresponds to the entrance of the cone in the experiment, it turns out that the results are insensitive to the point chosen. If, for instance, $x^{\prime}=-0.5$ were chosen as the starting point instead of $x^{*}=0$, the 
results would be nearly identical.

\section{Turbulence Spectra}

Spectra of $\bar{u}_{1}^{2 *}$ and $\bar{u}_{2}^{2}$ are plotted in figures 9 and 10 and show how contributions to $\bar{u}_{1}^{2}$ and $\overline{u_{2}^{2}}$ are distributed among dimensionless wave numbers (or reciprocal eddy sizes) $\kappa^{*}$. Plotted in this way the curve for no convergence or divergence $\left(\mathrm{x}^{\prime}=0\right)$ does not change with $x$. Thus, comparison of the curves for various values of $x^{*}$ with the curve for $\mathrm{x}^{\dagger}=\mathbf{0}$ shows how convergence or divergence affects the spectrum at a given position in comparison with the spectrum at the same position with no strain. For converging flow (negative $x^{*}$ ) the contributions to $\bar{u}_{1}^{*}$ occur at smaller wave numbers (larger eddies) than they would for no convergence, whereas contributions to $\bar{u}_{2}^{2^{*}}$ move to higher wave numbers. For diverging flow, by contrast, both $\psi_{11}$ and $\psi_{22}$ move to lower wave numbers than they would for no divergence.

Of some theoretical interest is the extreme asymmetry of the spectrum of $u_{1}^{2}$ for negatively large $x^{f}$. This effect has been observed previously (refs. 6 and 9), but it is much more pronounced here, possibly because the strain rate increases sharply as $x^{\prime}$ becomes more negative. As in the previous cases the asymmetry is associated with a spectral transfer term that depends on the mean strain rate. That the effect is associated with a transfer term (eq. (48)) was verified by solving the spectral equation with the transfer term omitted. The spectrum obtained was found to be nearly symmetrical.

A plot of the transfer term associated with the mean strain $T_{11}$ is given in figure 11. The net area under each curve is zero, in agreement with equation (46). The curve for negative $x^{\prime}$ is predominately negative at low wave numbers and positive at higher ones, so that energy is in general transferred from low wave numbers to higher ones. The curve also indicates that a small amount of reverse transfer to low wave numbers takes place in the very low wave number region.

The fact that the energy transfer in the longitudinal component of the turbulence is primarily from low to high wave numbers is possibly surprising, since according to a simplified theory it might be assumed that the $\overline{u_{1}^{2}}$ component is produced by vortices that are alined transversely. The axes of those vortices would tend to be shortened in a converging flow. However, the proportion of vortices alined in the transverse direction is small in a converging flow. Most of the contribution to $\overline{u_{1}^{2}}$ probably comes from oblique vortices, and in those vortices the energy transfer can be in the direction indicated in figure 11. For diverging flow (positive $x^{\prime}$ ) the energy transfer is in the opposite direc- 

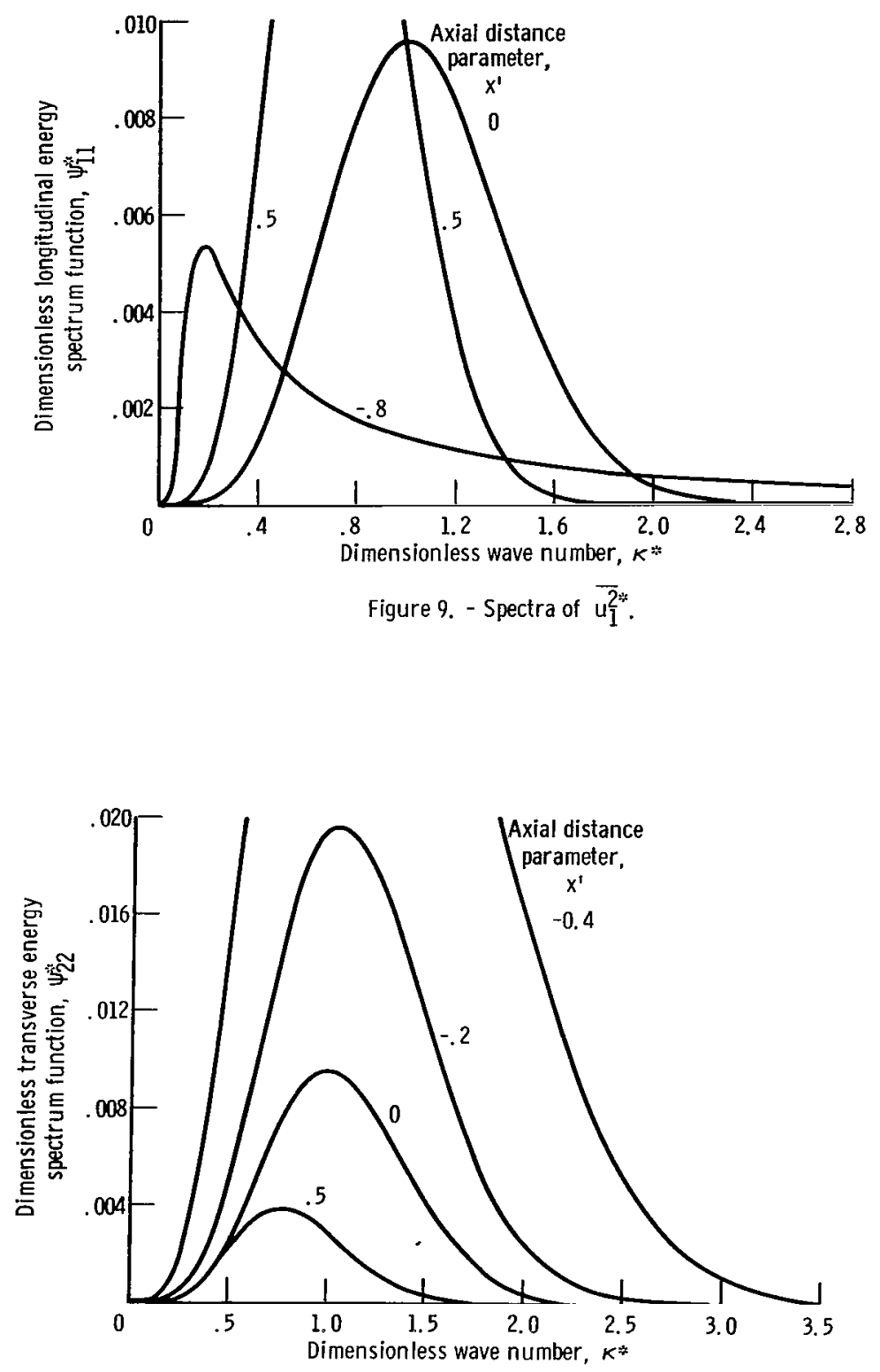

Figure 10. - Spectra of $\overline{u_{2}^{2}}$. 


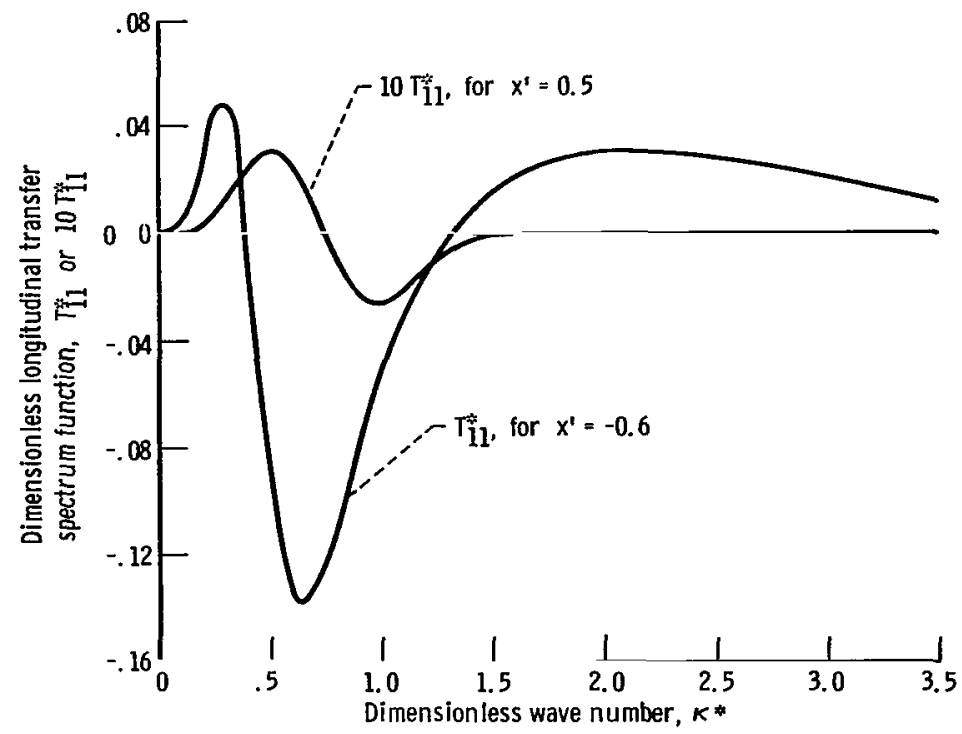

Figure 11. - Transfer spectra $T_{11}^{*}-X^{\prime}$ is an axial distance parameter.

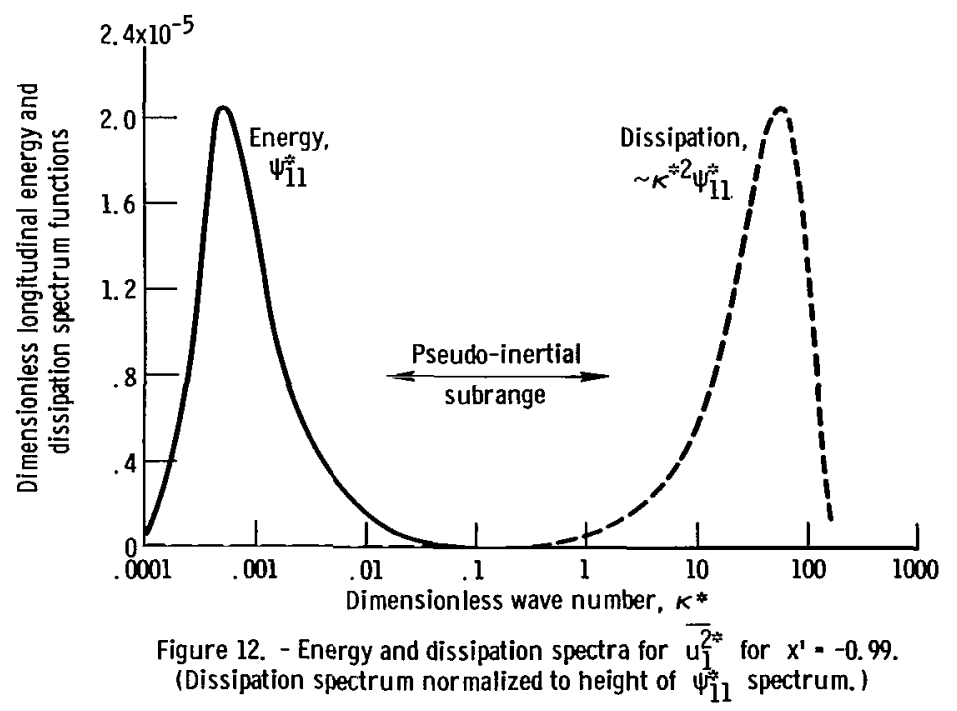

tion, that is, from high to low wave numbers. In that case the effect of the energy transfer on the shape of the $\overline{u_{1}^{2}}$ spectrum seems to be small.

For the converging case, figure 11 shows that the positive area in the high wave number region is spread out over a wide range of wave numbers. As $x^{\prime}$ becomes more negative this range widens still more. This elongated positive area of energy transfer is responsible for the long tail on the spectrum of $\overline{u_{1}^{2 *}}$. To carry the effect to the extreme, the energy spectrum of $\overline{u_{1}^{2}}$ for $x^{\prime}=-0.99$ is plotted semilogarithmically in figure 12. 
Included also in the plot is the dissipation spectrum, which is proportional to $\kappa^{2} \psi_{11}$. The energy and dissipation regions in this case show essentially complete separation and are separated by a pseudo-inertial subrange in which energy transfer is the dominating process. This inertial subrange is termed pseudo because it occurs only in one component of the turbulence and because it is produced by inertial effects associated with the mean strain rather than with the triple correlations that are usually considered to be responsible for an inertial subrange (ref. 8). Figure 12 is, however, a good illustration of a case in which essentially complete separation of energy and dissipation regions occurs.

Figure 13 shows a log-log plot of the spectrum of $\bar{u}_{1}^{2}$ for $x^{\prime}=-0.99$. In this plot $\psi_{11}^{*}$ is proportional to $\kappa^{*-1}$ over about four decades of $\kappa^{*}$. The region in which the curve begins to fall off more rapidly than $\kappa^{*-1}$ is roughly the region in which dissipation effects become important. For $x^{\prime}=-1$, the dissipation region would be moved to infinity and $\psi^{*}$ would vary as $\kappa^{*-1}$ over the entire range of wave numbers. The present results for a weak turbulence with large mean strain rates differ from the usual Kolmogorof $-5 / 3$ power spectrum that appears to apply at very high Reynolds numbers (ref. 14). As shown by the present results, however, a $-5 / 3$ power region in an energy spectrum is not necessary for complete separation of the energy and dissipation regions. In fact, any power between 0 and -2 will do as well, since the dissipation spectrum is obtained by multi-

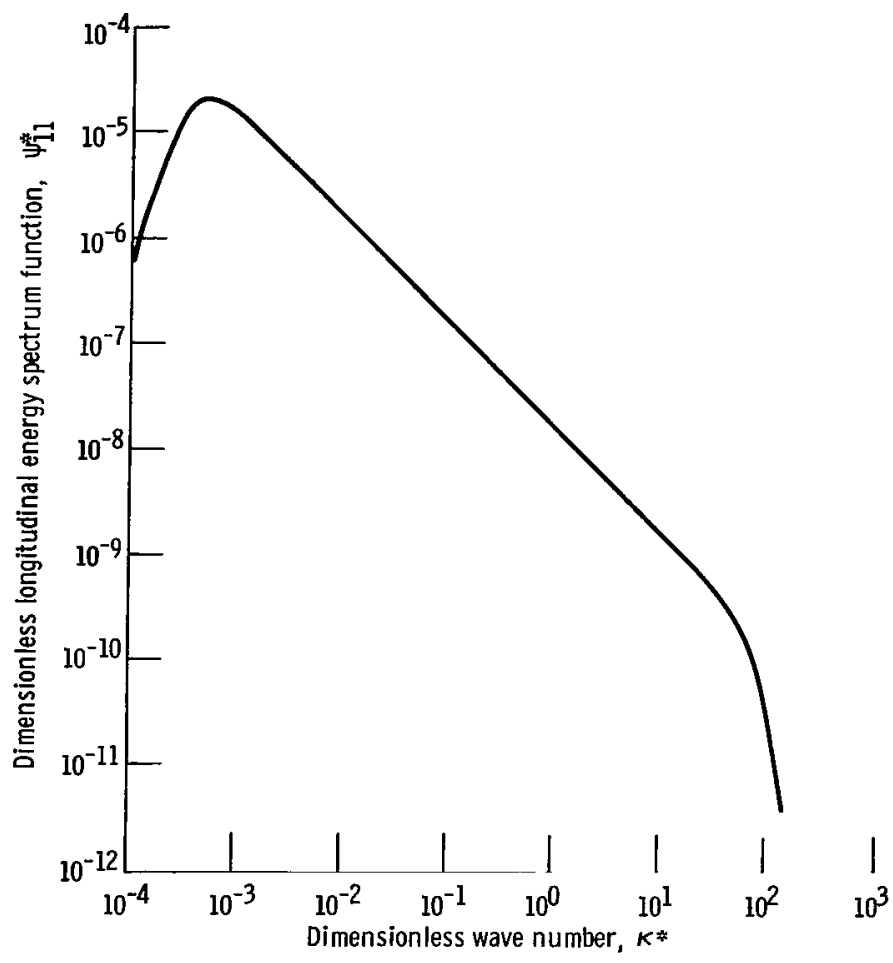

Figure 13. - Log-log plot of spectrum of $\overline{u_{1}^{2 *}}$ for $x^{\prime}=-0.99$. 


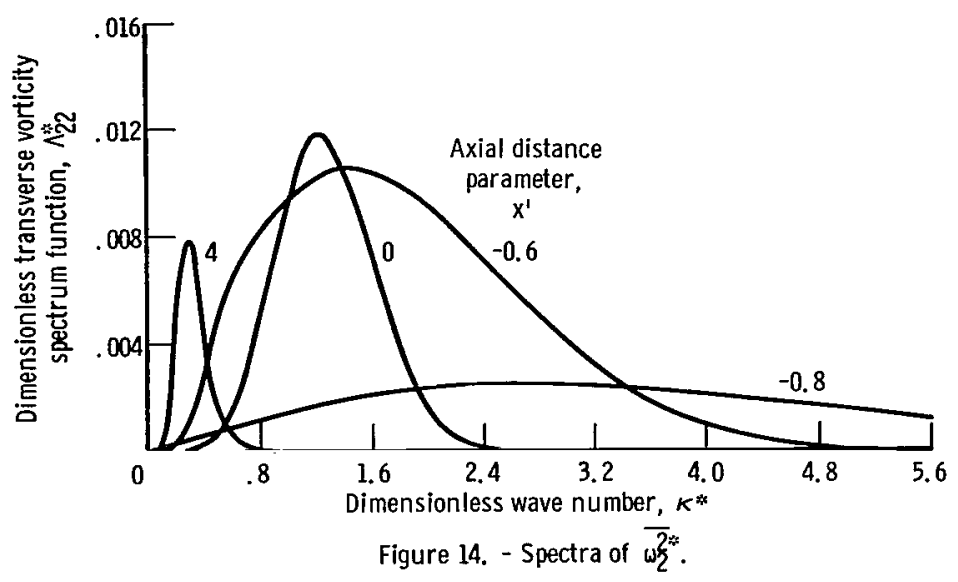

plying the energy spectrum by $\kappa^{2}$.

Vorticity spectra were also calculated, and representative results for spectra of $\bar{\omega}_{2}^{2}$ are plotted in figure 14. As $x^{\imath}$ becomes more negative, the spectra move to higher dimensionless wave numbers. Contributions to $\overline{\omega_{2}^{2}}$ also become spread out over a much wider range of wave numbers or vortex sizes. As $x^{*}$ increases positively, the spectra move to lower dimensionless wave numbers. The trends for the spectra of $\bar{\omega}_{1}^{2}$ (not shown) are similar to those shown in figure 14 with the exception that the shapes of the spectra do not change appreciably with strain. Thus, the vortices in converging flow tend to be smaller at a given $x$ than they would be for no convergence, whereas they tend to be comparatively large for diverging flow.

\section{SUMMARY OF RESULTS}

Near the virtual origin of the turbulence, for both converging and diverging flow through a cone, all turbulence components decreased along the flow because of viscous dissipation. For a converging flow the effect of the distortion was to tend to aline the turbulent vorticity in the direction of mean flow. This caused the longitudinal component of the velocity variance to decrease toward zero and the lateral components to increase. For a diverging flow the vorticity tended to be alined in the transverse direction. In that case the ratio of longitudinal to transverse velocity variance ultimately approached 5 . When the results for turbulent intensity were corrected to eliminate viscous decay and divided by local mean velocity, both longitudinal and transverse components decreased along a converging flow and increased along a diverging flow. The results were related approximately to heat transfer between a cone wall and a fluid and gave trends very 
similar to those observed experimentally.

Turbulent vorticity spectra showed that the turbulent vortices in a converging flow tend to be smaller at a given position than they would be for no convergence, whereas those in a diverging flow tend to be relatively large. A mean-strain transfer term in the spectral equation for the longitudinal component of the turbulence transferred energy in the high wave number direction for a converging flow. This transfer caused the spectrum for the longitudinal component of the energy to become strongly asymmetric. Near the cone apex essentially complete separation of the longitudinal energy and dissipation spectra was obtained. A power spectrum of -1 rather than $-5 / 3$ that extended over a considerable range of wave numbers was also noted for the longitudinal energy component. Thus, a $-5 / 3$ power spectrum is not a necessary consequence of the separation of the energy and dissipation regions.

Lewis Research Center,

National Aeronautics and Space Administration,

Cleveland, Ohio, June 9, 1966, 129-01-09-07-22. 


\section{APPENDIX - SYMBOLS}

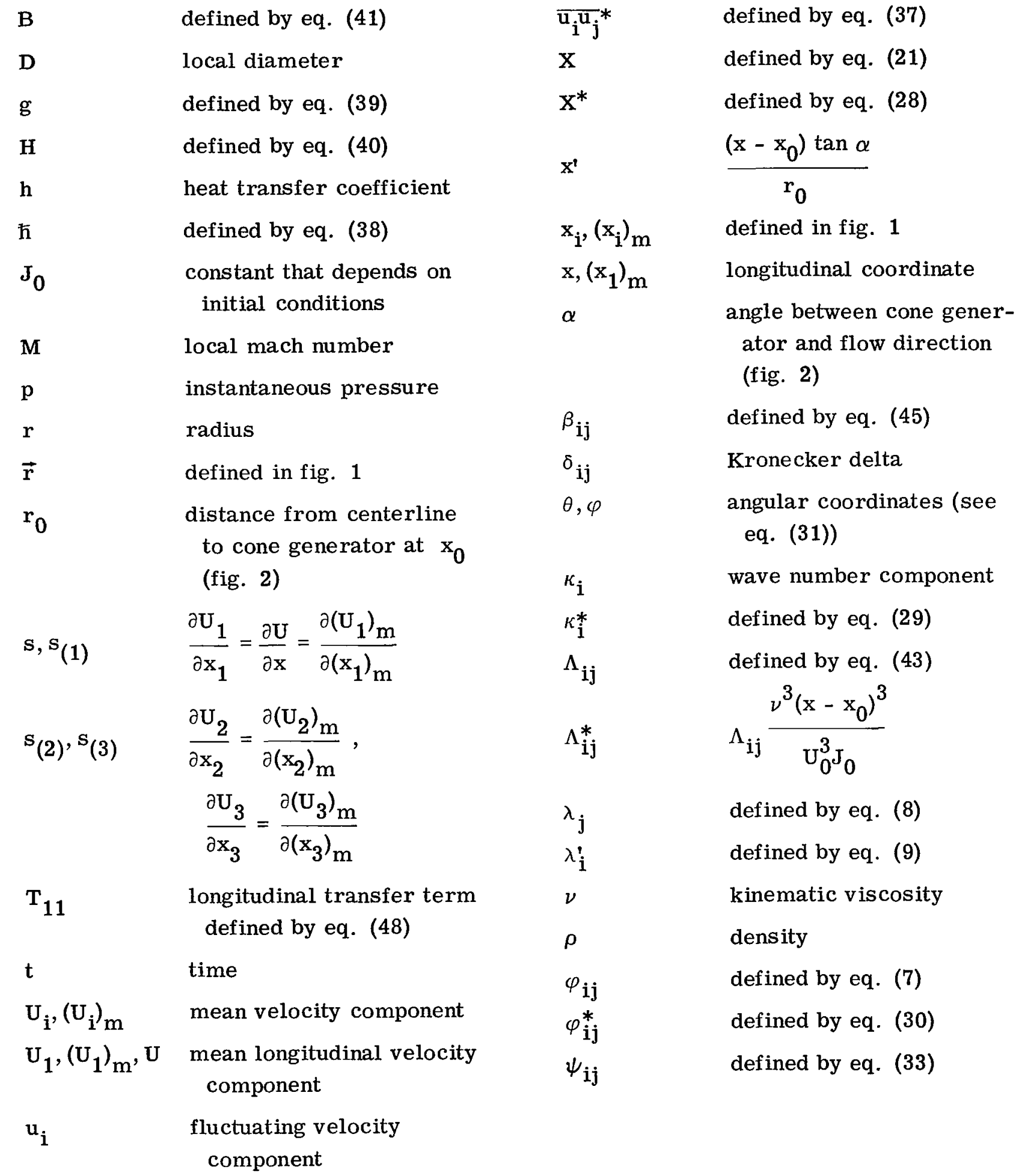


$\psi_{\mathrm{ij}}^{*} \quad \psi_{\mathrm{ij}} \frac{\nu^{2}\left(\mathrm{x}-\mathrm{x}_{0}\right)^{2}}{\mathrm{U}_{0}^{2} \mathrm{~J}_{0}}$

$\Omega_{i j} \quad$ defined by eq. (42)

$\overline{\omega_{\mathbf{i}} \omega_{\mathbf{j}}} \quad$ turbulent vorticity variance

$\bar{\omega}_{\mathrm{i}} \omega_{\mathrm{j}} * \quad \bar{\omega}_{\mathrm{i}} \omega_{\mathrm{j}} \frac{\nu^{7 / 2}\left(\mathrm{x}-\mathrm{x}_{0}\right)^{7 / 2}}{\mathrm{U}_{0}^{7 / 2} \mathrm{~J}_{0}}$

Subscripts:

a conditions upstream of contraction at point where mean velocity begins to vary (fig. 4)

C corrected to eliminate viscous decay

$\mathbf{m}$

at point $\mathbf{P}_{\mathrm{m}}$ (see fig. 1)
2,3

Superscripts:

*

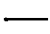

without effects of strain

at virtual origin of turbulence where turbulent energy would be infinite; it is assumed that turbulence is isotropic at $x_{0}$ and that strain begins to act there

in longitudinal direction

in transverse direction

at point $\mathrm{P}^{\prime}$ (see fig. 1), except for dimensionless $\mathrm{X}^{\prime}$

dimensionless quantity

averaged quantity 


\section{REFERENCES}

1. Prandtl, L.: Attaining a Steady Air Stream in Wind Tunnels. NACA TM 726, 1933.

2. Taylor, G. I. : Turbulence in Contracting Stream. Zeit. Angew. Math. Mech., vol. 15, no. 1/2, Feb 1935, pp. 91-96.

3. Ribner, H. S.; and Tucker, M.: Spectrum of Turbulence in a Contracting Stream. NACA TN 2606, 1952.

4. Batchelor, G. K. ; and Proudman, Ian: The Effect of Rapid Distortion of a Fluid in Turbulent Motion. Quart. J. Mech. Appl. Math., vol. 7, 1954, pp. 83-103.

5. Pearson, J. R. A. : The Effect of Uniform Distortion on Weak Homogeneous Turbulence. J. Fluid Mech., vol. 5, pt 2, Feb. 1959, pp. 274-288.

6. Deissler, Robert G. : Effects of Inhomogeneity and of Shear Flow in Weak Turbulent Fields. Phys. Fluids, vol. 4, no. 10, Oct. 1961, pp. 1187-1198.

7. Hildebrand, Francis B.: Advanced Calculus for Engineers. Prentice-Hall, Inc., 1949 , p. 374.

8. Batchelor, George K. : The Theory of Homogeneous Turbulence. Cambridge University Press, 1953.

9. Deissler, Robert G.: Effect of Uniform Longitudinal Strain Rate on Weak Homogeneous Turbulence in a Compressible Flow. NASA TN D-2800, 1965.

10. Mills, Robert R., Jr.; and Corrsin, Stanley: Effect of Contraction on Turbulence and Temperature Fluctuations Generated by a Warm Grid. NASA Memo 5-5-59W, 1959.

11. Uberoi, Mahinder S.: Effect of Wind-Tunnel Contraction on Free-Stream Turbulence. J. Aeron. Sci., vol. 23, no. 8, Aug. 1956, pp. 754-764.

12. Boldman, Donald R. ; Schmidt, James F.; and Fortini, Anthony: Turbulence, HeatTransfer, and Boundary Layer Measurements in a Conical Nozzle with a Controlled Inlet Velocity Profile. NASA TN D-3221, 1966.

13. Back, L. H. ; Massier, P. F. ; and Gier, H. L.: Convective Heat Transfer in a Convergent-Divergent Nozzle. Int. J. Heat Mass Transfer, vol. 7, no. 5, 1964, pp. 549-568.

14. Grant, H. L. ; Stewart, R. W. ; and Moilliet, A.: Turbulence Spectra from a Tidal Channel. J. Fluid Mech., vol. 12, pt. 2, Feb. 1962, pp. 241-268. 
"The deronautical and space activities of the United States sball be conducted so as to contribute. . . to the expansion of buman knowledge of pbenomena in the atmospbere and space. The Administration shall provide for the widest practicable and appropriate dissemination of information concerning its activities and the results thereof."

-National Aeronautics and Space Act of 1958

\section{NASA SCIENTIFIC AND TECHNICAL PUBLICATIONS}

TECHNICAL REPORTS: Scientific and technical information considered important, complete, and a lasting contribution to existing knowledge.

TECHNICAL NOTES: Information less broad in scope but nevertheless of importance as a contribution to existing knowledge.

TECHNICAL MEMORANDUMS: Information receiving limited distribution because of preliminary data, security classification, or other reasons.

CONTRACTOR REPORTS: Technical information generated in connection with a NASA contract or grant and released under NASA auspices.

TECHNICAL TRANSLATIONS: Information published in a foreign language considered to merit NASA distribution in English.

TECHNICAL REPRINTS: Information derived from NASA activities and initially published in the form of journal articles.

SPECIAL PUBLICATIONS: Information derived from or of value to NASA activities but not necessarily reporting the results of individual NASA-programmed scientific efforts. Publications include conference proceedings, monographs, data compilations, handbooks, sourcebooks, and special bibliographies.

Details on the availability of these publications may be obtained from:

SCIENTIFIC AND TECHNICAL INFORMATION DIVISION

NATIONAL AERONAUTICS AND SPACE ADMINISTRATION

Washington, D.C. 20546 